\title{
EDITORIAL
}

\section{What editors want in an abstract}

\author{
Catherine M Ketcham ${ }^{1}$, Robert W Hardy ${ }^{2}$, Brian Rubin ${ }^{3}$ and Gene P Siegal ${ }^{4}$ \\ Laboratory Investigation (2010) 90, 4-5; doi:10.1038/labinvest.2009.122
}

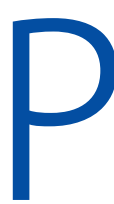

lenty of good advice is available on how to

write a quality abstract for a scientific paper. ${ }^{1-6}$ The main purpose of the abstract is to provide a concise and accurate summary of the research. Abstracts are generally written with the reader in mind, and rightly so. However, there is good reason for authors to keep the editors and reviewers in mind while writing abstracts. First impressions are important, and to be a successful contributor to Laboratory Investigation (LI), an author should provide a wellwritten and accurate abstract that makes it clear to the editors that a paper is within the scope of the journal, contains a complete complement of experiments with cohesive results to stand as a significant advance in the field, and is worthy of peer review. Furthermore, the abstract, standing alone, must also be able to entice busy reviewers to accept an invitation to critique the work.

The purpose of this editorial is not to ask authors to choose between catering to the editors and informing the end users; rather, it is to make the point that an abstract that pleases the editors and reviewers will also be appreciated by the scientists who see the final version of the paper.

\section{THE CONTENT OF AN ABSTRACT}

${ }^{1}$ University of Florida College of Medicine, Gainesville, FL, USA;

${ }^{2}$ Department of Physiology and Biophysics, University of Alabama at Birmingham, Birmingham, AL, USA; ${ }^{3}$ Cleveland Clinic Lerner College of Medicine of Case Western Reserve University, Cleveland, OH, USA and ${ }^{4}$ Department of Pathology, University of Alabama at Birmingham, Birmingham, AL, USA.

Correspondence should be addressed to: catherine@uscap.org
An abstract must be 'concise, lucid and well organized.' The general consensus is that it should be 200-300 words in length and should contain a one- to two-sentence introduction, a mention rather than a long description of the materials and methods, and the results as the bulk of the abstract. ${ }^{2-4,6,7}$ In order for the readers to have the proper perspective of the work, there should also be a statement of significance near the beginning or at the end of the abstract, and a one- to twosentence discussion at the very end. ${ }^{2}$ The readers should not have to refer to the paper to understand the abstract; ${ }^{5}$ it should contain everything that readers need and nothing that they do not need. ${ }^{7}$ We would argue it also needs a clearly stated purpose, goal, or hypothesis after the introduction and it needs a conclusion at the end that addresses that goal.

\section{ADVICE FROM THE EDITORS}

As LI has received manuscripts in which the abstracts hinder rather than help, the editors would like to offer specific advice on preparing an abstract that will enhance the chances of the manuscript progressing quickly through peer review. Although this editorial is somewhat specific to LI, many of the concepts can be applied to other scientific journals.

To understand the significance of the abstract to the LI editors, it is important to understand the journal's work flow. When a manuscript arrives on LI's web site, the Managing Editor (ME) reads the abstract and forms an opinion as to whether the paper is within the scope of the journal and consists of more than a 'least publishable unit' (LPU). The $\mathrm{ME}$ then examines the figures closely and skims the rest of the paper. The manuscript is then sent to the Editor-in-Chief or a Senior Associate Editor, along with the ME's notes. The editor repeats this exercise and makes the decision as to whether to advance the paper to an Associate Editor (AE) or reject it 'prior to review? The AE, almost always an expert in the paper's field, closely scrutinizes the manuscript. The $\mathrm{AE}$ either assigns potential reviewers or recommends rejection of the manuscript.

Thus, papers submitted to LI have to progress through three distinct tiers of editors to make it to peer review. When a request to review is sent out to potential referees, the message only contains the title and abstract; the entire paper is not available unless the invitee accepts the assignment. It follows that a clear, compelling and accurate abstract tends to help a paper gain the attention of the editors and attract the most appropriate reviewers in a timely manner.

The one general message the editors can offer authors is that it is undesirable for there to be any kind of disconnect between the abstract and the paper itself. Such inconsistencies could make a 
reviewer decline after starting the review, because the paper seemed be about one thing according to the abstract, yet turned out to be something else entirely. A poor abstract may even be a cause for rejection of the paper without peer review. Some specific advice to scientists considering publishing in LI follows:

State the hypothesis clearly and explain the importance of the topic. The working hypothesis needs to be made apparent early in the abstract, as does the justification for the study. If it is not quickly obvious what the authors are doing and why, there is a risk of having the paper rejected on the basis of priority.

Do not include too much introductory material. It is best not to squander too many of the 250 allowed words on introductory material; otherwise the editors could start wondering whether the paper is actually an unsolicited review article. The bulk of the text should be the results and conclusions of that manuscript.

A word about the methods. Though the methods section of an abstract is necessarily short, the editors and reviewers need to know several things about the experimental design in addition to the types of protocols used. It is very helpful to reveal whether the experiments were carried out with human tissue, animals or cell lines. If the study is confined to a specific organ system, it should be stated up-front, and the origin, including species, of any cell lines should be mentioned.

Avoid jargon and non-standard or undefined abbreviations. It is safe to assume that the LI editors know what DNA and PCR are, but, nevertheless, it is necessary to define most abbreviations. Even if everyone in the authors' specialty knows certain terminology, remember that the manuscript has to usually make it through at least two editors at LI before it gets to an expert in the authors' field.

All of the main conclusions of the paper need to be mentioned in the abstract. Authors sometimes shortchange themselves by not revealing all of the significant findings of the paper in the abstract; under these circumstances, a data-rich paper can be mistaken for less than one LPU and rejected as incremental. Conversely, it is misleading to ignore contradictory or negative data presented in the paper when writing the abstract. Again, the conclusion should address the hypothesis or purpose. If it does not, then the significance established in the first part of the abstract becomes irrelevant.

Do not make promises that are not delivered in the paper. Editors and reviewers especially dislike overselling the significance of the work in the abstract. Speculation masquerading as a finding is as inappropriate in the abstract as it is in the paper.

Make it obvious that the paper fits the scope of the journal. Authors should always determine that their paper fits within the scope of a journal before submitting. LI is a basic and translational pathology research journal and the editors are looking for papers that define the mechanisms of disease. Manuscripts appropriate for the journal sometimes have abstracts that appear to be less about pathology and more about biochemistry/ physiology/genetics/developmental biology. To avoid having such papers rejected as out of scope, it is a good idea to have a sentence in the abstract as to how the results of the paper relate to the mechanism (or pathobiology) of a disease.

If the manuscript has been changed, the abstract needs to be changed. Sometimes authors neglect to amend the abstract after a paper has been extensively revised, which results in major differences between the paper and the abstract. Always reevaluate the abstract after a paper has been through edits.

In conclusion, the abstract provides the author an opportunity to make a best first impression. The editors of LI are not suggesting that imperfect abstracts always doom papers that are otherwise worthy, or that stellar abstracts can save flawed studies. Rather, the editors believe that optimizing the abstract of a scientifically sound manuscript expedites its progression through peer review and will ultimately attract more attention to the published paper and its results.

\section{DISCLOSURE/CONFLICT OF INTEREST}

The authors declare no conflict of interest.

1. Wilkins R. Editorial: How to write an abstract. Circulation 1958;17:841.

2. Baillie J. On writing: write the abstract, and a manuscript will emerge from it!. Endoscopy 2004;36:648-650.

3. Perannnunzii L. How to write an original article for the Journal of Orthopaedics and Traumatology. J Orthopaed Traumatol 2008;9:1-3.

4. Curtis P. How to write a scientific. (abstract) http:// web3.uwindsor.ca/kits/jlichaa/59-110a/vck13materials.nsf/ 467dadf398d63ead8525676d005c149d/691b975a8dec382 f8525735a006fb05b/\$FILE/How\%20to\%20write\%20an\% 20abstract.pdf.

5. Barrett KE. How to write (and review) a scientific paper. http://74.125.95.132/search?q = cache:O6UUJOnA7XcJ: the-aps.org/careers/careers1/mentor/workshop/2001/ Barrett.doc+How+to+Write+(and+Review) + a+Scientific+ Paper+Kim+E.+Barrett,+Ph.D.\&cd $=3 \& \mathrm{hl}=$ en\&ct $=$ clnk\&gl = us.

6. De Smet AA, Manaster BJ, Murphy Jr WA. How to write a successful abstract. Radiology 1994;190:571-572.

7. Elefteriades JA. Twelve tips on writing a good scientific paper. Int J Angiol 2002;11:53-55. 